\title{
Research on the Effectiveness of Nutrition Supply in Beach Volleyball Playing Based on R Weighted Mean Algorithm
}

\author{
Xiu-yun Cui
}

Department of Physical Education, Xi'an University of Architecture and Technology, Xi'an 710055, China

\begin{abstract}
The special environment in beach volleyball playing generates intense stimulation on human bodies, which is pretty helpful in improving the performances of many sports. Meanwhile, the nutrition supply is also showing its critical significance. Due to the altitude environment, a special requirement is imposed on the calorie and nutrition supplied to the bench volleyball players in training. Therefore, the players should intake the sugar, sports drink, minerals, vitamins and some nutritional additives, correspondingly. At last, the R Weighted Mean Algorithm (RWMA) is used to analyze the data from the sample. The result shows that on the basis of the beach volleyball player's need for nutrition, we should give the beach volleyball player meals of high carbohydrate and other nutritional supplement. And this may have some help to the improving of the beach volleyball player's health and physical ability.
\end{abstract}

Keywords: Nutrition and food safety, Beach volleyball player, R Weighted Mean Algorithm (RWMA), Effectiveness.

\section{INTRODUCTION}

Beach volleyball, a new sport that was created in America in 1930s, has been the rage in 1970s. Especially after it had been placed on Olympic Games, rocketing development has made it to be the sport that is full of the most potential and the most sight in the modern field now.

In our country, the very beginning of introduction of beach volleyball was in July 1989. In recent years, as a new sport and with the initial stages of national sport industrialization which is a relative good environment for developing sports, beach volleyball do has some development, but it still leaves much to be desired even without negative impact of old sport management system.

In the beginning, the beach volleyball was played purely to satisfy people's entertainment and amusement, and then it became gradually preferred by the outdoor sports fans, and gradually swept the whole globe [1].

During the late 1980s and early 1990s, Beach Volleyball came to China. Although the time after its introduction was very short, it became very popular among college students. The reasons for this phenomenon includes: it's good for fitness, entertainment, fun, fashion; enlightening, and other functions; its venues are delicate, soft, effectively reducing sports injuries and trauma; it has fewer restrictions on the venue, suitable for different age, sex, levels of the participants, and having the various forms of competition: competition within the university, competition between the universities students tournaments, and so on.
At present, the "Outline of the Development of Volleyball Program-Beach Volleyball in 2001-2008" made by the Chinese Volleyball Association, specially emphasizes that Beach Volleyball program should strengthen its cooperation with the departments of education and promote the schoolbased youth beach volleyball projects including "promising project", "Seed Project", and "Hope Project" [2]. What's more, Beach Volleyball in 1996 was included in the Olympic Events, and in recent years the level of the Chinese women's volleyball team began to rebound, which once again aroused people's passion of volleyball. At this special time, can inject new blood and bring new vitality to Beach Volleyball program, too.

Researchers have explored the effect of different scoring systems and court dimensions in the sport [3]. Common findings in the literature suggested that the smaller court dimensions increased match duration and changed the physiological demands of the sport [Xiang, et al., 2005]. Similarly, skill sets used by winning teams also varied in accordance with a change in court dimension. With side8out scoring, a major difference between winning and losing teams was their ability to receive the serve successfully, whereas in rally point scoring, success was determined by kill efficiency [4]. At this point it is important to note that literature pertaining to playing characteristics in the sport of beach volleyball published before 2001 will show results that are inconsistent with the way that the modern game is played (personal discussion with Canadian national beach volleyball coach), [5].

In the modern beach volleyball match, the speed of the change between the two teams' attack and defend becomes faster and faster. And the match also becomes more and more intense. The beach volleyball player, as the core of the match, must suit the needs of the development of the beach volleyball match. So, they should take some special training of physical ability, and if they do not have excellent physical 
ability and nutrition supply, the beach volleyball player would not have good performance in the match.

The rapid and effective promotion and development of Beach Volleyball in China Universities has certain practical significance. Colleges usually have large sports population; the students have high cultural quality, strong sense of sport awareness, and strong power of publicity. Most of the students of Physical Education will work in the basic units, which can bring the awareness of Beach Volleyball and activity habits there, thus make this sport spread wholly. What's more, it can be conducive to select "promising talents", and cultivate the reserve forces of this sport, and gradually perfect the personnel training system, and ultimately promote popularizing and competitive levels of Beach Volleyball.

\section{THE CURRENT SITUATION OF DEVELOPMENT OF BEACH VOLLEYBALL PLAYING IN CHINA}

First, marketization of professional beach volleyball in China means that professional beach volleyball as a kind of sports service product enters the orbits of market economy and runs well guided by the market and centered by the economic benefits. In the marketing operation process, with sports economics, sports management and marketing management as the guidance, marketization of the professional project beach volleyball is summarized and operationally analyzed.

Secondly, professional beach volleyball market, in which nature of the product, investment and financing channels, the organization and management mode, the benefit are different from other sports market in China, is the emerging market in the process of development of the market economy. The formation, the objectives of the organization, the structure, the distribution of control rights and operation mechanism of marketization of professional beach volleyball and professional beach volleyball club system in China, are different from other developed countries' professional beach volleyball market.

Thirdly, the establishment of the market economy system and its development, and reformation of competitive sports in China promote the formation and development of the marketization of professional beach volleyball. Professional beach volleyball clubs become the main running bodies of the market; meanwhile, the government's support and competition system provide a platform of the formation of the marketization of professional beach volleyball. The Government Guiding supply pattern is currently "bottleneck" restriction of development of marketization of professional beach volleyball in China.

Fourthly, media, fans, sponsors and local governments are the main bodies of our professional beach volleyball market demand. Supply bodies are professional beach volleyball clubs and event organizations. Professional beach volleyball competition market intermediary agencies mainly undertake the business activities and projects promotion agency. Professional beach volleyball price mechanism mainly manifests that the prices of the products, the characteristics of the product, the influence factors of the pricing and selection pricing strategy, and etc.
Fifthly, during the market equilibrium of professional beach volleyball, the role of price factor is limited to supply and demand market balance. The main factor influencing the consumer demand in the market are consumer utility, product prices and consumer preferences, the capacity of sports promotion agency and the development and operation of intangible assets of Professional Beach Volleyball Club. Non-price factors have become elements of a balanced supply and demand. Professional beach volleyball has characteristics of market equilibrium as follows: the regional differences between the market equilibrium prices are very significant, and the status of market supply and demand trends in non-equilibrium state.

Sixthly, the relationship between professional beach volleyball tournament organizations and investors is fuzzy. Despite the principal-agent relationship in the form, it is lack of system and mechanism of contract and constraint. Compared with AVP professional beach volleyball in America, the current cooperation and contract in China is limited between professional beach volleyball tournament organizations and their investment operators, and the mechanisms are inadequate to establish the agency.

Seventhly, there are problems existing in our professional beach volleyball project market, for instance, the market scale, structure, operation mechanism and the internal and external market environment of. The main coping strategies are: accelerate and perfect the market system and the market, further improve the' national tour of the organizational structure, establish property system of the market main coping reasonably as soon as possible, create favorable policy environment, and formulate and improve the related laws and regulations.

The development strategy of competitive volleyball refers to system, stage, and scientific planning to the global development strategy of competitive volleyball and methods and strategies of coordinating the global. Chinese volleyball association's 2001-2008, 2009-2016 plan is the manifestation of the strategy of development of competitive volleyball in China. The implementation of the two plan states the strategy but there are many practical problems in the implementation of the plan. Resolution of these issues must proceed from the strategic height, through a comprehensive, longterm, integrated approach and strategy to solve.

The management model, athlete's cultural quality, volleyball Club, training effect, reserve training and coach's effect are the major restrictive factors affecting development of competitive volleyball in China in the new period. In these factors, perfecting management mode is to protect and improving coach's overall quaffed is the key, raising the level of sports team training is fundamental, and strengthen training reserve personnel is the foundation of, improving athletes' cultural quality is the driving force, and improving living environment of Club is a prerequisite.

The international promotion, competition systems, economic investment and business development is the promotion of competitive volleyball development in China in the new period. Among them, the rapid development of international Volleyball has provided the conditions for development and improvement of Chinese competitive volleyball, 
volleyball league implementation of host-guest-court system provides organizations to ensure raising the level of volleyball sports in China, China's rapid economic development provides material guarantee for the development of Chinese competitive volleyball, commercial development and sports professional model has provided experience for the development of Chinese Professional volleyball. According to the advantage and disadvantage, opportunity and threaten existing in athletic volleyball development during Chinese new era, there are four development strategies can be selected: pioneering strategy of playing internal advantage and using external opportunities of organization; protest type strategy of using organization advantage and avoiding or reducing its external threat; by of fighting strategy by using external opportunities to cover internal disadvantage of organization; and conservative type strategy by reducing internal disadvantage of organization and avoiding external environment threat. Each strategic development direction includes the two strategies types.

Therefore, the cooperation with various media should be strengthened. Propagandizing beach volleyball vigorously and the work of propagandizing should be focused on the particular culture meaning of beach volleyball. It should enlarge our nation's population of beach volleyball. The introduction of middleman into beach volleyball match market should be intensified based on reciprocal condition. The combination management of beach volleyball match with travel industry, entertainment industry, rag trade and cosmetic industry should be reinforced widely.

\section{THE R WEIGHTED MEAN ALGORITHM AND RESEARCH METHOD}

The detected impulses will be removed by $\mathrm{R}$ algorithm. Let $f_{i, j}^{\prime}$ be the value of the sample at statistics location $(i, j)$. For the corrupted statistics $(i, j)$, the sample size $\left(2 L_{f}+1\right) \times\left(2 L_{f}+1\right)$ is used. Starting with $L_{f}=1$, this sample size iteratively extends outward by one statistics in its four sides until the number of free statistics (denoted by $P_{i, j}$ ) within this sample size is not less than 1 . Let $W_{i, j}^{\prime}$ denote the values of free statistics in the concerned area, i.e.,

$$
\begin{array}{r}
W_{i, j}^{\prime}=\left\{f_{i+s, j+t}^{\prime} \mid b_{i+s, j+t}=0, b_{i, j}=1,\right. \\
\left.(s, t) \neq(0,0),-L_{f} \leq s, t \leq L_{f}\right\}
\end{array}
$$

The weighted mean value $g_{i, j}$ of the statistics values in $W_{i, j}^{\prime}$ is defined as:

$$
g_{i, j}=\frac{\sum_{f_{i s, j+t} \in W_{i, j}^{\prime}} w_{i+s, j+t} f_{i+s, j+t}^{\prime}}{\sum_{f_{i+s, j+t} \in W_{i, j}^{\prime}} w_{i+s, j+t}}
$$

)

where $w_{i+s, j+t}$ means the weight of $f_{i+s, j+t}^{\prime}$. Let $m_{i, j}^{\prime}$ be the median value of $W_{i, j}^{\prime}$. Because the median value has the least probability to be the value of the corrupted statistics [1], $m_{i, j}^{\prime}$ is utilized to determine $w_{i+s, j+t}$. It is easy to understand that the smaller the absolute difference between $f_{i+s, j+t}^{\prime}$ and $m_{i, j}^{\prime}$, the larger the weight $w_{i+s, j+t}$ should be to strengthen the influence of $f_{i+s, j+t}^{\prime}$ on $g_{i, j}$. Based on extensive simulations which indicate that $w_{i+s, j+t}$ is dependent on both above absolute difference and noise ratio, $w_{i+s, j+t}$ is chosen as:

$$
w_{i+s, j+t}=R+(1-R) \sqrt{\frac{\frac{\left|f_{i+s, j+t}^{\prime}-m_{i, j}^{\prime}\right|}{f_{\max }^{\prime}-f_{\min }^{\prime}}}{1-\frac{\left|f_{i+s, j+t}^{\prime}-m_{i, j}^{\prime}\right|}{f_{\max }^{\prime}-f_{\min }^{\prime}}}}
$$

Where $f_{\max }^{\prime}$ and $f_{\min }^{\prime}$ denote the maximum statistics value and the minimum one in the concerned area, respectively.

The output is obtained by:

$h_{i, j}=b_{i, j} \cdot g_{i, j}+\left(1-b_{i, j}\right) f_{i, j}^{\prime}+\Delta \omega_{i, j}$

Then we have:

$q_{i}=[x, y, z, \psi, \theta, \phi]_{i}^{T}, \quad q=\left[q_{1}^{T}, q_{2}^{T}, \ldots, q_{n}^{T}\right]^{T}$

The equation is established. The $\mathrm{R}$ weighted mean algorithm takes the Cartesian coordinates and Euler angle that respectively shows the position and direction of sample orient as the generalized coordinate, and establishes statistics equation by $\mathrm{R}$ weighted mean algorithm:

$$
\begin{aligned}
& \frac{d}{d t}\left(\frac{\partial T}{\partial \dot{q}}\right)^{T}-\left(\frac{\partial T}{\partial q}\right)^{T}+\phi_{q}^{T} \rho+\theta_{q}^{T} \mu=Q \\
& \text { Integrity Constraint Equation: } \\
& \phi(q, t)=0
\end{aligned}
$$

Non-Integrity Constraint Equation:

$$
\theta(q, \dot{q}, t)=0
$$

\section{THE NUTRITION SUPPLEMENT DURING BENCH VOLEYBALL PLAYING BASED ON THE EXPERI- MENT DATA}

The experiment group is trained with correct nutrition supplement in a bench volleyball playing game while the reference group is trained without correct nutrition supplement in a same bench volleyball playing. The experiment data is obtained and disposed by $\mathrm{R}$ weighted mean algorithm. The Fig. 1 shows comparison of the performance of the two groups. Each group is trained without the nutrition supplement for one hour, the experimental data is record. Then each group is trained with the nutrition supplement for one hour in a same bench volleyball playing, the experimental data is also record. The experiment data is obtained and disposed by $\mathrm{R}$ weighted mean algorithm. Fig. 2 shows the comparison of the performance before and after the nutrition supplement for each group [6-7].

While in the bench volleyball training, the players have to overcome both the hypoxia stimulation and large consumption of energies. In order to prevent the undue accumulation of fatigue caused by huge energy consumption, the body is 


\begin{tabular}{|c|c|c|c|c|c|c|c|c|c|c|}
\hline \multirow[t]{3}{*}{ ه } & \multicolumn{2}{|c|}{ Basic Techniques } & \multicolumn{2}{|c|}{ Ba sic Techniques } & \multicolumn{2}{|l|}{ Rules } & \multicolumn{2}{|c|}{ Tea ching Method } & \multicolumn{2}{|c|}{ Average $(\%)$} \\
\hline & Experiment & Reference & Experiment & Reference & Experiment & Reference & Experiment & Reference & Experiment & Reference \\
\hline & Groups & Group & Group & Group & Group & Group & Group & Group & Group & Group \\
\hline Excellent & 16 & 18 & 19. & 60 & 15 & 50 & 25 & 213 & 19.7 & 12.7 \\
\hline Good & 670 & 450 & 550 & 360 & 67 & 430 & 580 & 480 & 62.70 & 430 \\
\hline Pass & 14 & 320 & 230 & 580 & 150 & $48+3$ & 9 & 160 & 16.2 & 38.20 \\
\hline Fail & 0 & 50 & 0 & 0.0 & 0 & 40 & 50 & 150 & 1.20 & 40 \\
\hline Total & 970 & 1000 & 970 & 1000 & 970 & 1000 & 970 & 1000 & حـ & ه \\
\hline
\end{tabular}

Fig. (1). The Comparison of the Performance of the Two Groups.

\begin{tabular}{|c|c|c|c|c|c|c|c|c|c|c|c|c|c|c|}
\hline 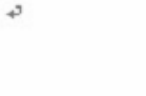 & & $\begin{array}{l}\text { Team } \\
\text { spinit }\end{array}$ & $\begin{array}{l}\text { Team } \\
\text { duty }\end{array}$ & $\begin{array}{c}\text { Cooperation } \\
\text { behavior }\end{array}$ & $\begin{array}{l}\text { Obedience } \\
\text { behavior }\end{array}$ & $\begin{array}{l}\text { Devotion } \\
\text { behavior }\end{array}$ & $\begin{array}{l}\text { Self } \\
\text { value }\end{array}$ & $\begin{array}{l}\text { Leader } \\
\text { behavior }\end{array}$ & $\begin{array}{c}\text { Competition } \\
\text { sense? }\end{array}$ & $\begin{array}{l}\text { Self } \\
\text { confidence }\end{array}$ & $\begin{array}{l}\text { Innovative } \\
\text { e behavior }\end{array}$ & $\begin{array}{l}\text { Rule } \\
\text { senses }\end{array}$ & $\begin{array}{c}\text { Rule } \\
\text { behavior }\end{array}$ & Note \\
\hline Experiment & Befores & 590 & 59 & 45 & 320 & 360 & 830 & 500 & 280 & 340 & 390 & 380 & 370 & $\checkmark$ \\
\hline groups? & After & 850 & 850 & 890 & 580 & 560 & 830 & 76 & 800 & 76 & 78 & 870 & 870 & $\checkmark$ \\
\hline \multirow{2}{*}{$\begin{array}{l}\text { Reference } \\
\text { groups }\end{array}$} & Befores & 41 & 350 & 43 & 290 & 290 & 710 & 510 & 29 & 380 & 350 & 590 & 590 & $\checkmark$ \\
\hline & After & 400 & 36 & 590 & 330 & 290 & 680 & 530 & 350 & 400 & 320 & 680 & 680 & $\checkmark$ \\
\hline
\end{tabular}

Fig. (2). The Comparison of the Performance Before and After the Nutrition Supplement For Each Group.

required to supplement some carbohydrates which consume little oxygen but release energies quickly.

In the adaptive period, the content of fat should be reduced and carbohydrate increased. Supplement of glucose before exercise can increase the glycogen store in the body; supplement of glucose during the exercise can effectively maintain the blood sugar level and defer the fatigue; and that after the exercise if in favor of the composition and recovery of muscle glycogen and hepatic glycogen. In addition, the moderate supplement of sugar can stimulate the secretion of insulin, enhance the composition of muscle protein and improve the immunity of the body. During the supplement of sugar, attention should be paid to the category of the sugar as well as the time and concentration of the sugar supplemented. The common sugar includes the glycogen, fructose and oligosaccharide, of which the honey is a kind of natural fructose. In the meantime, the easy-to-digest food is advised to be input. Commonly, the concentration of sugar supplement is better be between $5 \%$ and $10 \%$, and that it could be a little higher after the exercise, between $10 \%$ and $35 \%$. The bench volleyball players need to consume more sugar in the altitude training than in the plain training. This sugar is mainly acquired from the food and beverage containing sugar. Therefore, the food must contain the high-concentration sugar, which is also easy to absorb.

According to the latest study conducted by University of Connecticut, the anti-resistance training in the dehydrated state is pretty unpromising, as it would has a passive influence on the hormone state which is favorable to muscle growth. The researchers let the participant undertake the same training content in three states, which are normal state, dehydrated state (reduction of $2.5 \%$ weight) and extremely dehydrated state (reduction of 5\% weight). Then, they extracted the participant's blood to measure the change of the hormone related to the muscle growth, such as the testosterone, cortisol, growth hormone, Para insulin growth factor, insulin, glucose and lactate, etc. It was founded that the common state has two passive effects on the testosterone: one of them is to raise the level of cortisol which would fight the testosterone for the receptor, thus lower the level of the testosterone; the other is that it reduce the total amount of the testosterone due to the exercise. In addition, this state also has influent on the metabolism of the carbohydrate and fat, which means that people will have a higher content of body fat. The sports beverage has the following functions: a) promoting drinking; b) rapidly recovering and maintaining the body fluid equilibrium; c) supplying energy and improving the sports ability. In the meantime, the following conditions also need more attention.

The higher the concentration of the electron cut in and sugar in the sports drink is, the higher the osmotic pressure will be, and that will result in the reduction of drain of beverage in the stomach. As the electron cut in concentration and osmotic pressure of in the sweet is higher than in the blood plasma, the loss of water is more than the cut in in the blood when the sweet is secreted quickly.

Therefore, the supplement sports drink should be a low osmotic pressure or equilibrium pressure, i.e. 250-370 units 
of the osmotic pressure. The sugar content of the sports drink should be between $4 \%$ and $8 \%$, and the sugar could be the glucose, cane sugar, oligosaccharide, short-chain starch (such as maltose dextrin) and so on. The oligosaccharide, often composed of 3-8 monosaccharide molecules, is absorbed slower than the monosaccharide and disaccharide, so it can prolong the time of sugar supply in the endurance exercise.

As a little sodium salt contained in the drink is good for absorption of the sugar and water, sports drinks often contain some, of which the concentration is lower than that in the blood, with the sodium concentration being about 20-60 $\mathrm{mol} / \mathrm{L}$. According to relevant studies, the intake of the sports drink with good tastes is obviously higher than fresh water. Generally, it's not expected to drink the carbohydrate sports drink before or during the exercise, as this kind of drink may generate the flatulence in the stomach.

The most suitable temperature is between $50{ }^{\circ} \mathrm{C}-130$ ${ }^{\circ} \mathrm{C}$.Apart from the function of reducing the body temperature, it can also create a good flavor which is helpful in intake. During the training, the mineral water can be chosen plus glucose $3156 \mathrm{~g}$, sodium chloride $0147 \mathrm{~g}$, potassium chloride $0130 \mathrm{~g}$ and sodium citrate buffer $0153 \mathrm{~g}$. While supplementing the sports drink, the players should adopt the principle of "many times, little amount". Besides, the urine proportion and weight would better be recorded to monitor the volume of the sports drink.

When undergoing the bench volleyball training, the supplement of trace elements and minerals is effective in getting rid of tiredness and muscle spasm as well as maintain the water-salt balance in human bodies. 1) During the bench volleyball training, the nutritional reserve including the iron is very important to athletes' competence. On the plateau, the athletes' hematopoietic function is obviously enhanced, for example, the red blood cell and hemoglobin are remarkably increased. Therefore, during the bench volleyball training, the iron element is helpful in raising the content of the hemoglobin and oxygen in the blood. During the period of bench volleyball training, the players should not only eat the food rich in iron, such as the animal organ, egg yolk, bean and fish, but also appropriately supplement some iron preparations which are slightly stimulating to the stomach and intestine. The specific operation is as follows: have the liquid iron preparation 3 times per day (total volume for the male is $200-250 \mathrm{mg}$ per day and for the female $100-150 \mathrm{mg}$ ). Shortage in iron during the training will lead to the sports anemia, which not only harms the oxygen carrying capacity, but also damages the athlete's health, while the excessive iron may be toxic. The iron supplement should be conducted under the doctor's guidance. Before the training, the iron reserve in the body must be at the normal level. 2) The copper $(\mathrm{Cu})$ is one of the necessary trace elements needed by all organisms. With the copper, the iron can't enter the hemoglobin cell during the biological synthesis mechanism. Therefore, lack of copper also leads to anemia, even if there is sufficient iron. A plain athlete must intake 2-4 mg of copper. However, the copper can't be taken too much, as it has antagonism effect with the zinc. The food that is richest in copper includes the animal giblet, shellfish (oyster), nut, bean, raisin and drupe (apricot). 3) Zinc is needed by all organisms, as it is the component of more than 200 metal enzymes. The food that is rich in zinc includes the fresh meat, liver, egg and marine organism (oyster and menhaden). A plain athlete needs to supplement $15 \mathrm{mg}$ of zinc, and the figure could be increased for those engaging bench volleyball trainings. 4) During the bench volleyball training, supplement of certain amount of potassium $(\mathrm{K})$, sodium $(\mathrm{Na})$ and magnesium $(\mathrm{Mg})$ is also needed, as these minerals along with others help the body afford the high-strength and huge-amount training and prevent the spasm caused by long-term, high-strength sports. During the training of large amount of exercise, the sweet and excretion may lead to the large amount of mineral loss, thus resulting in the loss of elements including $\mathrm{K}, \mathrm{Na}$ and $\mathrm{Mg}$. Therefore, the moderate supplement of these minerals is the assurance of the success of the bench volleyball training.

During the bench volleyball training, the metabolism of vitamins is also fastened. The study indicates that increase of Vitamin B (VBI, VB2, VB12 and VB15) in diet promotes human body to adapt to the plateau environment. According to a study conducted by Russian researchers, the moderate intake of VB 15 can increase the oxidative metabolism velocity of the tissue and improve the stability of the body in the hypoxia condition. Generally, the athlete would better take in $150 \mathrm{mg}$ of VB15 per day from one week before the bench volleyball training, and this is done continually in the whole training period.

After the adaptive phase of the training, double of the volume should be taken in due to the huge amount of training, thus ensuring the stability of the body. Vitamin E has the function of weakening the aggregation of platelets, which means it can lower the thickness of the blood and make more free iron in the blood. For the blood circulation, it's helpful to mediate the growth of the blood thickness caused by the increase of red cells during the bench volleyball training. During the bench volleyball training, 400 international units of vitamin E should be supplemented per day. The insufficient intake of the vitamin would affect the whole metabolism of the organism, lower the athletic ability and reduce the competence of adapting to the outdoor environment and resisting the illness. During the bench volleyball training, the supplement of the vitamin can not only improve the anoxia tolerance of the body, but also improve the function of the higher nervous activity, buffer the tiredness, and improve the sports tolerance and immunity of the body, thus making the athletes take part in the training in the optimal status. It is widely known that Vitamin D can strengthen the bone and have a lot of benefits on health.

In addition, the study conducted by Purdue University, the USA, founded that Vitamin B can also increase the heat effect of a meal. The recent study on the plateau proves that the athlete doesn't lack vitamin C; however, considering the vitamin $\mathrm{C}$ has the chemical function of promoting the redox process in the body, accelerating hematopoiesis and detoxifying, etc., moderate amount of vitamin $\mathrm{C}$ should be supplemented in the load training so as to lower the oxidative stress caused by the training.

\section{CONCLUSION}

Nowadays, with the competition standard and biological technology increasingly rising, the supplement of the sports 
nutrition provides an important support for the athletes to obtain good performances in the beach volleyball training. During the beach volleyball training, we would not only make appropriate training plans for athletes, but also formulate suitable dinning plans and develop the food and sports tonics rich in special nutrients specially for athletes, thus helping them to achieve ideal sports performances in the future competitions.

\section{CONFLICT OF INTEREST}

The authors confirm that this article content has no conflicts of interest.

\section{ACKNOWLEDGMENT}

This work is supported by the Key Project of Guangxi Social Sciences, China (No.gxsk201424), the Education Science fund of the Education Department of Guangxi, China (No.2014JGA268), and Guangxi Office for Education Sciences Planning, China (No.2013C108).

\section{REFERENCES}

[1] C. Ji, and C. Wang, "Influence of Physical Education Culture of Higher Learning Schools on the Personalities and Social Adaptation of Students," Journal of Guangzhou Physical Education Institute, vol. 26, pp. 116-119, 2006.

[2] L.E. Burke, and J. Fair, "Promoting prevention: skill sets and attributes of health care providers who deliver behavioral interventions," Journal of Cardiovascular Nursing, vol. 18, pp. 256-266, 2013.

[3] M.B. Potter, J.D. Vu, and M. C. Minihane, "Weight management: What patients want from their primary care physicians," The Journal of Family Practice, vol. 50, pp. 513-518, 2011.

[4] X. Wang, and J. Sun, "Analysis of issues related to development of beach volleyball in colleges and universities in China," Journal of Tianshui, vol. 29, pp. 100-102, 2009.

[5] Q. Yan, and P. Gong, "Investigation on the Nutrition Knowledge Attitudes and Dietary Behaviors of University Students," Modern Preventive Medicine, vol. 3, pp. 520-524, 2008.

[6] X. Xiang, Z. Yang, and Z. Miao, "Beach volleyball reserve personnel training mechanism and influence factors of ECG," Journal of Physical Education, vol. 12, pp. 117-119, 2005.

[7] Q. Zhang, S. Zeng, L. Zhu, and Z. Cai, "Nutritional Knowledge, Attitudes and Practices of Shantou University Students", Chinese Journal of School Health, vol. 5, pp. 412-413, 2001. 